\title{
Closed-form Force Distribution for Parallel Wire Robots
}

\author{
Andreas Pott, Tobias Bruckmann and Lars Mikelsons
}

\begin{abstract}
This paper presents an algorithm to determine feasible force distributions for parallel wire robots in closed-form. The force distributions are continuous along trajectories and differentiable at most of the points. The computational efforts are strictly bounded and small even for large numbers of wires. The algorithm is compared to other approaches for calculation of force distribution in terms of the numerical effort and their applicability for control purposes.
\end{abstract}

\section{INTRODUCTION}

Parallel wire robots are able to achieve very high velocities and accelerations due to very small inertia. Furthermore, large workspace and high payloads are possible due to the efficient force transmission through the wires. In the last decade, a lot of research has been carried out to study both theory (see e.g. [1, 4, 5, 6, 12]) and implementation $[2,7]$ of these robots.

For a mobile platform with $n$ degrees-of-freedom, in general, at least $m=n+1$ wires are required to fully control the motion [12]. Therefore, many wire robots are over-determined with respect to forward kinematics and under-determined with respect to distribution of forces in the wires (Fig. 1). As a consequence, a major issue in control is to calculate feasible force distributions in realtime. Furthermore,

Andreas Pott

Fraunhofer Institute for Manufacturing Engineering and Automation IPA, 70569 Stuttgart, Germany, e-mail: andreas.pott@ipa.fhg.de

Tobias Bruckmann

Chair of Mechatronics, University of Duisburg-Essen, 47048 Duisburg, Germany, e-mail: tobias.bruckmann@uni-due.de

Lars Mikelsons

Chair of Mechatronics, University of Duisburg-Essen, 47048 Duisburg, Germany e-mail: lars.mikelsons@uni-due.de 


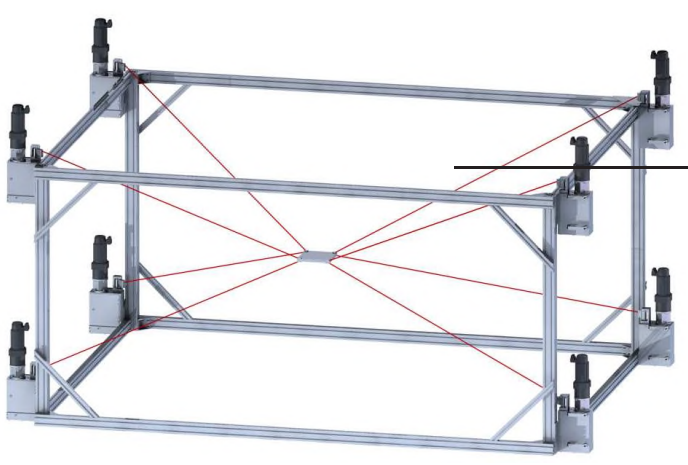

a)

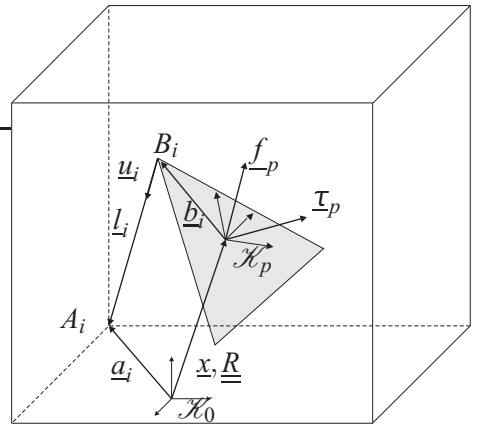

b)

Fig. 1: a) CAD draft of the spatial wire robot IPAnema with eight wires and six degrees-of-freedom. b) Geometry and kinematics of a general wire robot

reasonable workspace definitions include the condition to identify feasible force distributions at all poses within the workspace. Since this evaluation has to be performed repeatedly to calculate the workspace (based on checking the workspace on a number of points), also in this setting an efficient scheme is required, especially when it is used for robot design.

In this paper we propose an efficient closed-form solution for the problem of identifying and calculating force distributions for spatial wire robots. The application of the algorithm is straightforward even if the number of wires is relatively large (e.g. $m=12$ ) compared to the degrees-of-freedom.

This paper is organized as follows: Sec. 2 briefly recalls the well-known kinematic foundation of wire robots. In Sec. 3 the closed-form solution for force distribution is derived and analyzed. Results from control are presented in Sec. 4 and the paper closes with the conclusion in Sec. 5 .

\section{KINEMATIC FOUNDATION}

For better reference, the kinematic basics of wire robots are briefly reviewed $[1,6$, 12]. Fig. $1 \mathrm{~b}$ shows the kinematic structure of a spatial wire robot, where the vectors $\underline{a}_{i}$ denote the proximal attachment points on the frame, the vectors $\underline{b}_{i}$ are the relative positions of the distal attachment points on the movable platform, and $\underline{l}_{i}$ denote the length of the wires. Applying a vector loop, the closure-constraint reads

$$
\underline{l}_{i}=\underline{a}_{i}-\underline{x}-\underline{R}_{i} \quad \text { for } \quad i=1, \ldots, m
$$

where the vector $\underline{x}$ is the Cartesian position of the platform and the rotation matrix $\underline{\underline{R}}$ represents the orientation of the platform. The unit vector along the wire becomes 
$\underline{u}_{i}=\underline{l}_{i}|| \underline{l}_{i}||_{2}^{-1}$. For force equilibrium it holds $[9,12]$

$$
\underbrace{\left(\begin{array}{ccc}
\underline{u}_{1} & \cdots & \underline{u}_{m} \\
\underline{b}_{1} \times \underline{u}_{1} & \cdots & \underline{b}_{m} \times \underline{u}_{m}
\end{array}\right)}_{\underline{\underline{A}} \underline{\underline{x}}, \underline{\underline{R}})^{\mathrm{T}}} \underbrace{\left(\begin{array}{c}
f_{1} \\
\vdots \\
f_{m}
\end{array}\right)}_{\underline{f}}+\underbrace{\left(\begin{array}{c}
\underline{f}_{p} \\
\underline{\underline{\tau}}
\end{array}\right)}_{\underline{\underline{w}}}=\underline{0},
$$

where $\underline{f}_{p}, \underline{\tau}_{p}$ are the applied forces and torques, respectively, acting on the platform and $f$ is the vector of the wire forces. The matrix $\underline{A}^{\mathrm{T}}$ is the transpose of the Jacobian and referred to as structure matrix. It allows to investigate existence and quality of the workspace.

\section{FORCE DISTRIBUTION}

The determination of feasible force distribution for wire robots is a major challenge for design and control of such robots. The basic problem is to find positive solutions for the structure equation

$$
\underline{A}^{\mathrm{T}} \underline{f}+\underline{w}=\underline{0} \quad \text { with } \quad 0<f_{\min } \leq f_{i} \leq f_{\max }, \quad i \in[1, m]
$$

where $\underline{\underline{A}}^{\mathrm{T}}$ is the pose dependent structure matrix, $f$ collects the forces in the wires, and $\underline{w}$ are the forces and torques applied to the mobile platform including inertia and gravity. $f_{\min }$ denotes the minimum force in the wires e.g. to tense it and the maximal load $f_{\max }$ is due to limitations in the actuator or the breaking load of the wire. One has to distinguish two problems regarding force calculations for wire robots:

1. Is there at least one solution $f$ to Eq. (3) (does the given pose belong to the workspace)?

2. How to determine such solutions which are continuous along a trajectory (e.g. for force control purposes)?

In this work, a new method is presented to solve both problems for wire robots with an arbitrary redundancy $r=m-n>0$ of wires, so-called redundantly restrained positing mechanisms RRPM [9]. Verhoeven [12] and Gosselin [4] showed that one can find trajectories with continuous force distributions if one converts the problem into an optimization problem using $p$-norms

$$
\begin{array}{cl}
\underset{\text { minimize }}{g(\underline{f})=}\|\underline{f}\|_{p}=\sqrt{\sum_{i=1}^{m} f_{i}^{2}} \\
\text { subject to } & f_{\min } \leq f_{i} \leq f_{\max } \\
\text { linear constraints } & w_{i}=-\sum_{j=1}^{m} A_{j, i}^{\mathrm{T}} f_{j} .
\end{array}
$$


Some iterative procedures to solve this general problem have been proposed $[2,12]$. Especially for realtime control purposes, non-iterative methods with an acceptable amount of computation time were developed. Based on a barycentrical approach, their computation time is driven by triangulations in the $r$-dimensional space [8] which become more complex for higher dimensions. Currently, the barycentrical algorithm has been shown for redundancies up to $r=m-n=2$.

In this work, it is shown how to calculate a solution for this problem in closedform for $p=2$ with simple algebraic operations making an implementation in a realtime system straight forward.

\subsection{Force distribution in closed-form}

In order to find solutions, the wire force vector $\underline{f}$ is split into

$$
\underline{f}=\underline{f}_{m}+\underline{f}_{v}
$$

where $\underline{f}_{m, i}=\left(f_{\min }+f_{\max }\right) / 2$ is the mean feasible force distribution and $\underline{f}_{v}$ is an arbitrary force vector. Thus, one can rewrite Eq. (3)

$$
\underline{\underline{A}}^{\mathrm{T}} \underline{f}_{v}=\underbrace{-\underline{w}-\underline{A}^{\mathrm{T}} \underline{f}_{m}}_{\underline{b}} .
$$

It follows from Verhoeven's theorem [12], that a force distribution is continuous along a trajectory of the mobile platform if it is as close to the vector $\underline{f}_{m}$ as possible using a $p$-norm with $(2 \leq p<\infty)$. A similar definition of an optimal solution is [8]: In terms of reliability, a solution between the force limits $f_{\min }$ and $f_{\max }$ is desired to stay away from the critical force limits as far as possible, resulting in an average wire tension level. This assumption is a reasonable compromise between the minimum and maximum wire force level. The idea of the proposed method is to transform the problem using Eq. (7) such that one has to determine bounded, least-square solutions rather than searching for positive solutions, since the latter turned out to be a challenging problem.

Here, the Euclidian-norm $(p=2)$ is used to determine the least-square solution of the equation, which exactly fulfills Eq. (3) and has a minimum two-norm (Euclidian norm) with respect to $f_{m}$. This can be done by means of the MoorePenrose generalized inverse which is defined for matrices with more columns than rows as $\underline{\underline{A}}^{+\mathrm{T}}=\underline{\underline{A}}\left(\underline{\underline{A}}^{\mathrm{T}} \underline{\underline{A}}\right)^{-1}$. Multiplying $\underline{\underline{A}}^{+\mathrm{T}}$ from the left hand side of Eq. (8) gives $\underline{f}_{v}=-\underline{\underline{A}}^{\mathrm{T}}\left(\underline{w}+\underline{\underline{A}}^{\mathrm{T}} \underline{f}_{m}\right)$ and substituting this into Eq. (7) yields

$$
\underline{f}=f_{m}-\underline{A}^{+\mathrm{T}}\left(\underline{w}+\underline{A}^{\mathrm{T}} \underline{f}_{m}\right) \text {. }
$$

Finally, the resulting force distribution $f$ must be checked to be compliant with the force limits. The force distribution has the following features: 
- It satisfies exactly the structure equation (3).

- It satisfies Verhoeven's theorem, i.e. if $\underline{\underline{A}}^{\mathrm{T}}$ and $\underline{w}$ are continuous along a trajectory, then the computed $f$ is also continuous.

- $f$ can be computed explicitly, where for the numerical computation only the operations matrix inverse, matrix transpose and matrix multiplication are needed. Thus, computation time is strictly bounded allowing for use in realtime control.

- Feasibility $\left(f_{i} \in\left[f_{\min }, f_{\max }\right]\right)$ can be checked straight forward.

- The algorithm fails if $\underline{\underline{A}}^{\mathrm{T}} \underline{\underline{A}}$ is singular. This can be easily detected while inverting this matrix and such poses do not belong to the workspace due to rank deficit of the structure matrix $\underline{\underline{A}}^{\mathrm{T}}$.

\subsection{Existence and Uniqueness of the Force Distribution}

Note, that the algorithm may also fail to find a feasible force distribution between the force limits although such a distribution exists. Three cases can be distinguished (Fig. 2) based on

$$
\underline{f}_{v}=\underline{f}-\underline{f}_{m}=-\underline{\underline{A}}^{+\mathrm{T}}\left(\underline{w}+\underline{\underline{A}}^{\mathrm{T}} \underline{f}_{m}\right) .
$$

Case 1: If $\left\|\underline{f}_{v}\right\|_{2}<r_{i}=\frac{1}{2}\left(f_{\max }+f_{\min }\right)$, then a feasible distribution exists and is bounded by the minimum and maximum forces in each wire. Furthermore, under this condition the force distribution is differentiable along a differentiable trajectory and wrench $w$.

Case 2: If $\left\|f_{v}\right\|_{2}>r_{o}=\frac{1}{2} \sqrt{m}\left(f_{\max }+f_{\min }\right)$ then no feasible force distribution exists and the pose does not belong to the workspace for the given applied wrench $\underline{w}$.

Case 3: Elsewise the algorithm might fails to find a feasible solution although it exists. In case 3 a no solution exist, thus it cannot be found. In case $3 \mathrm{c}$ a feasible solution outside of sphere $r_{i}$ is found. Although there are feasible solutions in case 3 b (see Fig. 2), the algorithm determines a solution outside the feasible region. Currently, we have no simple criterion to distinguish between $3 \mathrm{a}, 3 \mathrm{~b}$, and $3 \mathrm{c}$, but the algorithm is robust in that way that if it rather misses an existing solution than suppling an invalid solution.

To study the unique domains where the algorithm guarantees to determine the solution, tt is interesting to compare the volume of the inner $m$-hypersphere, the outer $m$-hypersphere, and the hypercubes given by the force limits. The results of this comparison are quite non-intuitive for higher number of wires $m$. In general the volume $V_{m}$ of a $m$-dimensional sphere with radius $r$ is

$$
V_{m}=\frac{\pi^{\frac{m}{2}}}{\Gamma\left(\frac{n}{2}+1\right)} r^{m}
$$

where $\Gamma$ denotes Euler's Gamma-function. Fig. 2 b shows the relative volume of the enclosing sphere (case 2) and inner sphere (case 1). One can see from the diagram that the distinction of cases between $3 \mathrm{a}, 3 \mathrm{~b}$, and $3 \mathrm{c}$, becomes an important issue. 


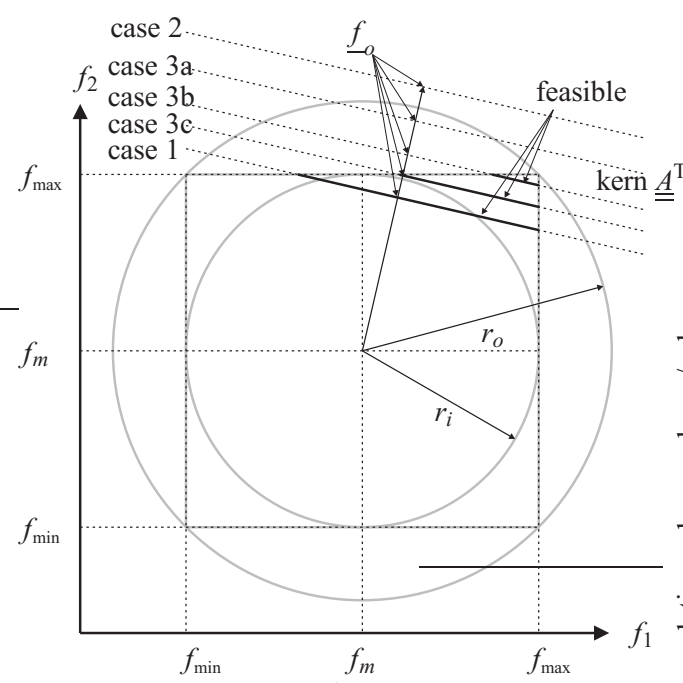

a)

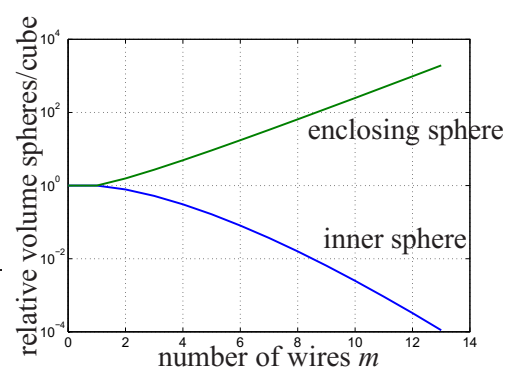

b)

Fig. 2: a) Determination of feasible force distributions b) Comparison of the volume of inner and enclosing $m$-spheres with the cube

\subsection{Implementation Issues and Performance}

Using the wire force $f$ determined from Eq. (9) one can answer the important questions of existence and value of the optimal solution by simply checking if it is feasible, i.e. $f \in\left[f_{\min }, f_{\max }\right]$. Thus, Eq. (9) renders a closed-form solution to the problem which only involves matrix multiplications, matrix transpose, and one matrix inversion. Thus, the computation time is well defined and strictly bounded. The latter is very important for applications in realtime control. Especially for a high number of wires, the computation is relatively cheap.

For implementation in an algorithm, one has to carefully check, if the inverse $\left(\underline{\underline{A}}^{\mathrm{T}} \underline{\underline{A}}\right)^{-1}$ exists. Physically speaking, this inverse exists if the robot is in a nonsingular configuration. Mathematically speaking, this can be checked if $\underline{\underline{A}}^{\mathrm{T}}$ has full row-rank.

A major advantage of the presented algorithm is its efficiency for higher degrees of redundancy $r=m-n$. Since Eq. (9) presents the solution for any $r>0$ one can investigate the computational efforts for different $r$. The number of operations to evaluate Eq. (9) is driven my the matrix multiplications and the matrix inversion. Thus, the overall effort is $\mathscr{O}\left(n^{2} m\right)+\mathscr{O}\left(n^{3}\right)$ and since in general the degree-of-freedom for robots is bounded by $n \leq 6$, the complexity for the algorithm is only $\mathscr{O}(m)$. This reveals that the runtime for force calculation is acceptable also for highly redundant robots. 


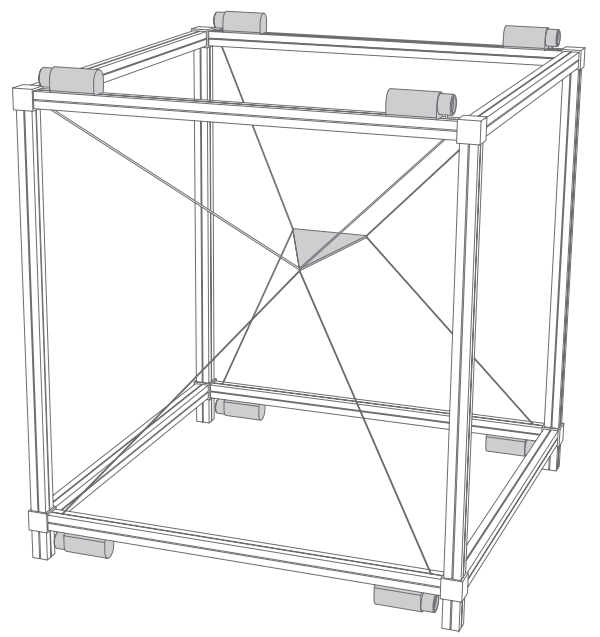

Fig. 3: The Segesta prototype

\section{RESULTS}

In this chapter, the presented algorithm is evaluated and compared to other known approaches. As a result, this may provide a first guideline for selecting a force calculation for a specific purpose.

In the past, different approaches were introduced to calculate force distributions, and each one of them delivers force distributions with different characteristics while requiring different computational effort, see [1] for an overview:

- Optimizer approach using a $p$-norm $[2,11,12]$

- Barycentric approach [8]

- Closed-form solution (presented here)

- Linear programming [10]

In the following sections, the barycentric approach as well as the optimizer approach are compared to the closed-form solution. It is reasonable to evaluate the methods by applying them to the real-time control system of the prototype SEGESTA.

The methods listed above are profiled with respect to their application on a realtime system. At the Chair for Mechatronics, University of Duisburg-Essen, the prototype SEGESTA was developed during the last decade $[3,6]$. Meanwhile, it is equipped with $m=8$ wires, directly driven by electronic commutated motors. To ensure a reliable operation of the system, a realtime control system DS1005 by dSPACE (PowerPC 705, $480 \mathrm{MHz}$ ) is used which is programmed in the MATLAB/Simulink language. The geometrical properties of the SEgESTA prototype (Fig. 3) are given in Tab. 1.

To get an overview regarding the performance of the force calculation methods under consideration, they are compared using the screw-shaped trajectory pictured 
Table 1: SEGESTA's geometrical parameters: platform vectors $\underline{b}$ and base vectors $\underline{a}$

\begin{tabular}{ccc}
\hline wire $i$ & platform vector $\underline{b}_{i}$ & base vector $\underline{a}_{i}$ \\
\hline 1 & {$[-0.0525,-0.0760,0.0]^{\mathrm{T}}$} & {$[0.0,0.0,0.0]^{\mathrm{T}}$} \\
2 & {$[-0.0525,-0.0760,0.0]^{\mathrm{T}}$} & {$[0.0,0.0,1.0]^{\mathrm{T}}$} \\
3 & {$[0.0525,-0.0760,0.0]^{\mathrm{T}}$} & {$[0.83,0.0,1.0]^{\mathrm{T}}$} \\
4 & {$[0.0525,-0.0760,0.0]^{\mathrm{T}}$} & {$[0.83,0.0,0.0]^{\mathrm{T}}$} \\
5 & {$[0.0,0.1240,0.0]^{\mathrm{T}}$} & {$[0.83,0.63,0.0]^{\mathrm{T}}$} \\
6 & {$[0.0,0.1240,0.0]^{\mathrm{T}}$} & {$[0.0,0.63,1.0]^{\mathrm{T}}$} \\
7 & {$[0.0,0.1240,0.0]^{\mathrm{T}}$} & {$[0.0,0.63,0.0]^{\mathrm{T}}$} \\
8 & {$[0.0,0.1240,0.0]^{\mathrm{T}}$} & {$[0.83,0.63,1.0]^{\mathrm{T}}$} \\
\hline
\end{tabular}

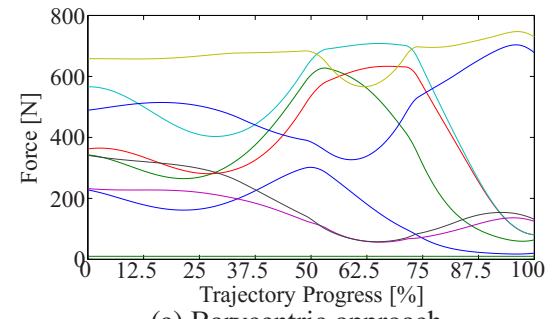

(a) Barycentric approach

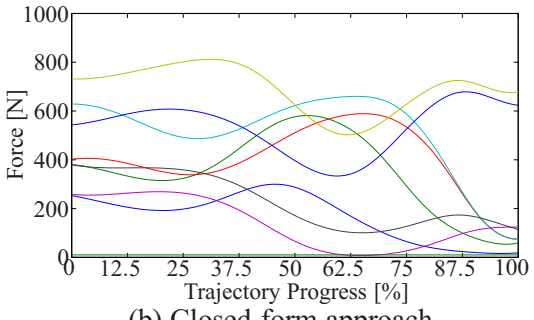

(b) Closed-form approach

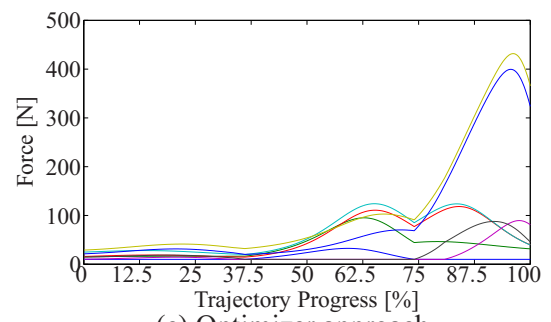

(c) Optimizer approach

Fig. 4: Force distributions along the shown screw-shaped trajectory using different force calculation algorithms

in Fig. 5. As shown in Fig. 4c, the optimizer approach delivers continuous solutions along the whole trajectory. Furthermore, these force distributions lead to a relatively low tension-level in the wires, i.e. they are optimal in the sense of minimality. The barycentric approach also (Fig. 4a) delivers feasible continuous force distributions along the whole trajectory, but the computed force distributions lead to a much higher tension level in the wires, i.e. the force distributions are optimal in the sense of maximizing the distance to the force limits. The closed-form approach delivers force distributions (Fig. 4b) that look very similar to the tensions calculated by the barycentric approach, but it fails where the path lies very close to the workspace boundary. This is an example for the case $3 \mathrm{~b}$, i.e. a feasible solution exists, but is 


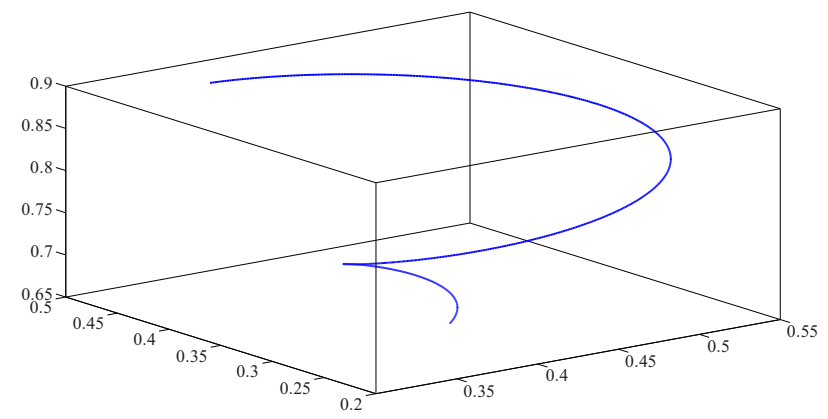

Fig. 5: Screw-shaped trajectory in the workspace of SEGESTA

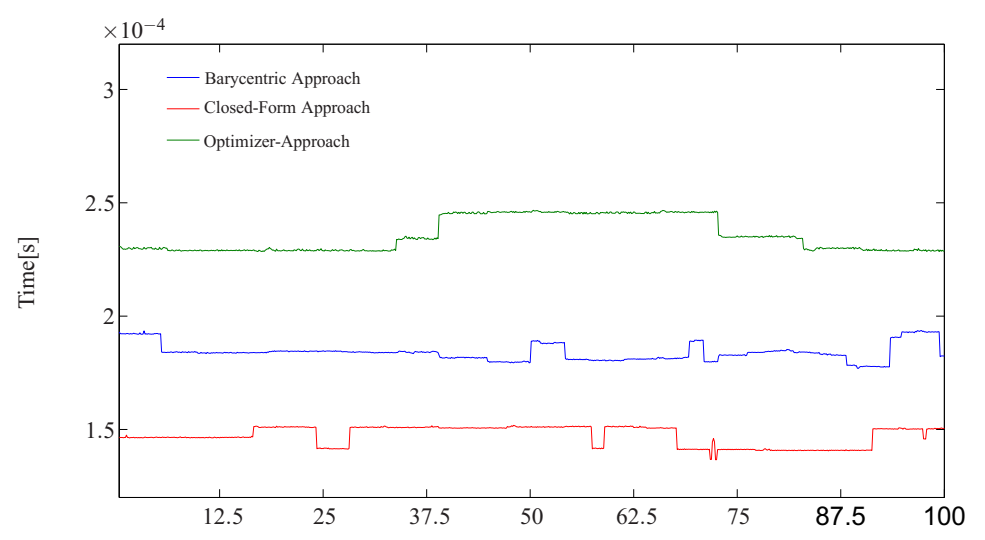

Fig. 6: Computation time with dSPACE system

not found since it lies outside the inner sphere. The calculation times are given in Fig 6. Therefore, the closed-form force calculation is suitable for realtime control as long as the end effector remains inside the (restricted) workspace, which is covered by the closed-form approach. In this case the closed-form approach is superior to the others in terms of calculation time. This holds especially for high redundancies, where the barycentric approach tends to be slow, due to the triangulation in high dimensions. The usage of the optimizer approach is dangerous in realtime control for all redundancies due to the - in general - non predictable worst case runtime. 


\section{CONCLUSION}

In this paper a new closed-form solution for the distribution of force for parallel wire robots is presented. This algorithm is capable to be applied for robots with arbitrarily many wires. Different properties are investigated with respect to the areas of application. For realtime force control of wire robots the calculation time of the algorithm is strictly bounded. Furthermore, the algorithm leads to continuous solutions along a trajectory. In a certain region, the solution is even differentiable. Contrary, the algorithm can be used in workspace determination. Interestingly, the presented formula reveals the influence of applied loads on the determined force distribution. The introduced approach is very fast and simple to implement.

\section{References}

1. Tobias Bruckmann, Lars Mikelsons, Thorsten Brandt, Manfred Hiller, and Dieter Schramm. Wire robots part i - kinematics, analysis and design. In Parallel Manipulators - New Developments, ARS Robotic Books, Vienna, Austria, 2008. I-Tech Education and Publishing.

2. Tobias Bruckmann, Andreas Pott, and Manfred Hiller. Calculating force distributions for redundantly actuated tendon-based Stewart platforms. In Advances in Robot Kinematics, pages 403-412, Ljubljana, Slovenia, 2006. Springer-Verlag.

3. Shiqing Fang. Design, Modeling and Motion Control of Tendon-Based Parallel Manipulators. Fortschritt-Berichte VDI, Reihe 8, Nr. 1076. VDI Verlag, Düsseldorf, 2005.

4. Clément Gosselin. On the determination of the force distribution in overconstrained cabledriven parallel mechanisms. In Proceedings of the Second International Workshop on Fundamental Issues and Future Research Directions for Parallel Mechanisms and Manipulators, pages 9-17, Montpellier, France, 2008.

5. M. Gouttefarde, J.-P. Merlet, and D. Daney. Wrench-feasible workspace of parallel cabledriven mechanisms. In IEEE International Conference on Robotics and Automation, pages 1492-1497, Roma, Italy, 2007.

6. Manfred Hiller, Shiqing Fang, Sonja Mielczarek, Richard Verhoeven, and Daniel Franitza. Design, analysis and realization of tendon-based parallel manipulators. Mechanism and Machine Theory, 40(4):429-445, April 2005.

7. Jean-Pierre Merlet and David Daney. A new design for wire-driven parallel robot. In $2 n d$ Int. Congress, Design and Modelling of mechanical systems, 2007.

8. Lars Mikelsons, Tobias Bruckmann, Dieter Schramm, and Manfred Hiller. A real-time capable force calculation algorithm for redundant tendon-based parallel manipulators. In IEEE International Conference on Robotics and Automation, Pasadena, 2008.

9. Aiguo Ming and Toshiro Higuchi. Study on multiple degree-of-freedom positioning mechanism using wires (part 1) - concept, design and control. Int. Journal of the Jap. Soc. for Precision Engineering, 28(2):131-138, June 1994.

10. S. R. Oh and S. K. Agrawal. Cable suspended planar robots with redundant cables: Controllers with positive tensions. In IEEE Transactions on Robotics, 2005.

11. J. A. Snyman and A. M. Hay. Analysis and optimization of a planar tendon-driven parallel manipulator. In Advances in Robot Kinematics, pages 303-312, Sestri Levante, Italy, 2004. Kluwer Academic Publishers.

12. Richard Verhoeven. Analysis of the Workspace of Tendon-based Stewart Platforms. PhD thesis, University of Duisburg-Essen, 2004. 